\title{
Linc00963: A novel, long non-coding RNA involved in the transition of prostate cancer from androgen- dependence to androgen-independence
}

\author{
LIJUAN WANG, SUXIA HAN, GUIHUA JIN, XIA ZHOU, MENG LI, \\ XIA YING, LE WANG, HUILI WU and QING ZHU \\ Department of Oncology, the First Affiliated Hospital, Xi'an Jiaotong University \\ of Medical College, Xi'an 710061, P.R. China
}

Received January 11, 2014; Accepted March 4, 2014

DOI: $10.3892 /$ ijo.2014.2363

\begin{abstract}
Whole genome transcriptomic analyses have identified a large number of long non-coding RNAs (lncRNAs), many of which are involved in a variety of biological functions. However, their functions and molecular mechanisms associated with prostate cancer (PCa) progression to a virulent and androgen-independent (AI) form remain elusive. Herein, we investigated the lncRNA expression profiles of the indolent, androgen-dependent (AD) LNCaP cell line to the aggressive metastatic, AI C4-2 cell line using microarray technology. The differentially expressed lncRNAs and genes were identified by microarray technology and the association in cis or in trans was analyzed to find potential lncRNA target genes. Expression of candidate lncRNAs and putative targets was evaluated by real-time quantitative reverse-transcription polymerase chain reaction (qRT-PCR). The functions of linc00963 on cell proliferation, apoptosis, migration and invasion were evaluated by a knockdown strategy in vitro using MTT, flow cytometric analysis and transwell chamber assays. IncRNAs $(\mathrm{n}=134)$ were differentially expressed (FDR $<0.001$ and fold change $\geq 2$ ) between the LNCaP and C4-2 cell lines. Linc00963 was upregulated most obviously evaluated by qRT-PCR. Knockdown of linc00963 attenuated C4-2 cell proliferation, motility, invasion ability, the expression of EGFR and phosphorylation levels of AKT, and promoted cell apoptosis. Linc00963 was involved in the prostate cancer transition from androgendependent to androgen-independent and metastasis via the EGFR signaling pathway.
\end{abstract}

Correspondence to: Professor Qing Zhu, Department of Oncology, the First Affiliated Hospital, Xi'an Jiaotong University of Medical College, Xi'an 710061, P.R. China

E-mail: newzhuqing1972@yahoo.com

Key words: long non-coding RNA, Linc00963, castration-resistant prostate cancer, epidermal growth factor receptor, AKT

\section{Introduction}

Prostate cancer ( $\mathrm{PCa})$ is the second leading cause of male cancer-related death in the United States, with 238,590 estimated new cases that occurred in 2013 and almost 29,720 deaths (1). Localized PCa patients have a long-term survival due to the combination of radiation and androgen deprivation therapy (2). However, clinical observation shows that after an initial responsiveness to androgen withdrawal treatment, almost all PCa will inevitably progress to recurrent castrationresistant prostate cancer (CRPC), and acquires the potential of metastasis, for which few therapeutic options with limited durability are available (3). The underlying mechanisms during the transition from androgen-dependent (AD) to androgenindependent (AI), from localized status to metastasis, remain to be elucidated. Identification of genes involved in this transition may provide insight into finding novel therapeutic strategies for CRPC.

Epidermal growth factor receptor (EGFR) is a member of the human epidermal growth factor receptor or ErbB family of receptor tyrosine kinases (4). EGFR is overexpressed in $40-80 \%$ of prostate cancer cells, and overexpression more common in African American men with prostate cancer (5). Furthermore, previous clinical studies suggested a correlation of EGFR expression with androgen-independence (6). More importantly, EGFR itself may be under the regulation of androgen signaling pathway, being negative in normal prostate cells but positive in prostate cancer cells, especially in androgen-independent cancer cells (7). In an effort to overcome castration-resistance, trials combining EGFR or dual kinase inhibitors with other novel agents are in development (8).

Emerging evidence indicates that LncRNAs which are RNA specis $>200$ bp in length (9) and frequently polyadenylated (10), are involved in physiological aspects of cell-type determination and tissue homeostasis (11), and in cancer lncRNAs are known to play important roles in carcinogenesis and tumor progression (12-16). One of the overexpressed lncRNAs in prostate cancer, PCGEM1, is tissue specific and PCa-associated lncRNA gene (15), whereas another highly expressed lncRNA, PRNCR1 (PCAT8), is pervasively transcribed from the critical region of 8q24 Region 2, which is 
significantly associated with PCa susceptibility (17). Previous studies also demonstrated that a novel long non-coding RNA (named PlncRNA-1) was frequently overexpressed in PCa cell lines and tissues and associated with cell viability and cell apoptosis (18). Recent studies have revealed the contribution of other lncRNAs as proto-oncogenes, drivers of metastatic transformation and tumor suppressor genes in prostate cancer, such as prostate cancer gene 3 (PCA3), metastasis associated in lung adenocarcinoma transcript 1 (MALAT-1), and PCAT-1 (13,19-21). However, the global expression profile of IncRNAs in androgen-dependent (AD) to androgen-independent (AI) prostate cancer is not fully uncovered.

Prostate cancer cell line LNCaP and C4-2 have the same genetic background and the unique advantage of remarkably mimicking the phenotypic and genotypic changes observed in clinical human PCa (22). In the current study, we used microarray technology to compare the IncRNA and mRNA expression between LNCaP cell line which was an androgen-dependent, non-metastasis (23) and the lineage-related C4-2 cell line which acquired characteristic of androgen-independence and possessed the capacity of metastasizing to lymph nodes and bone (24). As a result, $257 \mathrm{PCa}$-associated IncRNA transcripts were identified to be differently expressed in $\mathrm{LNCaP}$ and $\mathrm{C} 4-2$, including one well-known lncRNA, PlncRNA-1 (18). Among them, we further identified long intergenic non-protein coding RNA 963 (Linc00963) as the IncRNA with the most significantly different expression in LNCap and C4-2 cell lines using real-time PCR. Additionally, we identified EGFR as the putative target molecule by bioinformatics prediction. We further showed that suppression of Linc00963 by siRNA could reduce the capacity of cell viability and invasion and the expression of EGFR in C4-2 cells in vitro.

In the present study we demonstrated for the first time that Linc00963 was significantly associated with the capacity of cell metastasis in prostate cancer cells, and EGFR was the putative target molecule of Linc00963. Taken together, our data suggested that Linc00963 plays an important role in the transition from $\mathrm{AD}$ to $\mathrm{AI}$, and it could be a useful therapeutic target to prevent $\mathrm{PCa}$ metastasis.

\section{Materials and methods}

Cell culture. $\mathrm{LNCaP}$ and $\mathrm{C} 4-2$ cell lines were cultured in RPMI-1640 (Invitrogen, Carlsbad, CA, USA) supplemented with $8 \%$ fetal bovine serum (FBS; Hyclone, Logan, UT, USA), $10 \mathrm{mM}$ HEPES, $1.0 \mathrm{mM}$ sodium bicarbonate and $1 \%$ antibiotic/antimycotic solutions. All the cells were cultured at $37^{\circ} \mathrm{C}$ in a humidified atmosphere of $5 \% \mathrm{CO}_{2}$.

RNA extraction and microarray target preparation. Total RNA was extracted from C4-2 and LNCaP cell lines using TRIzol reagent (Invitrogen) according to the manufacturers' protocols. RNA cleanup including a DNase I digestion step was performed using RNeasy spin columns (Qiagen). RNA integrity was measured by the relative abundance of $28 \mathrm{~S} / 18 \mathrm{~S}$ ribosomal subunits, verified through micro-fluid capillary electrophoresis (Agilent Bioanalyzer 2100).

Microarray analysis. For microarray analysis, qualified total RNA was further purified by RNeasy mini kit (Qiagen) and
RNase-free DNase set (Qiagen). Total RNA was then amplified and labeled by Low Input Quick Amp Labeling kit (Agilent), following the manufacturer's instructions. Labeled cRNA were purified by Qiagen RNeasy ${ }^{\circledR}$ mini kit. Each Slide was hybridized with $600 \mathrm{ng}$ Cy3/Cy5-labeled cRNA using Gene Expression Hybridization kit (Agilent) in Hybridization Oven (Agilent), according to the manufacturer's instructions. After $17 \mathrm{~h}$ of hybridization, slides were washed in staining dishes (Thermo Shandon) with Gene Expression Wash Buffer kit (Agilent). Slides were scanned with Agilent C Scanner Settings, Dye channel: Green, scan resolution $=3 \mu \mathrm{m}, 20$ bit; Red, scan resolution $=5 \mu \mathrm{m}, 20$ bit. Data were extracted with Feature Extraction software 10.7 (Agilent). Raw data were normalized by Quantile algorithm, Gene Spring Software 11.0 (Agilent). Hierarchical clustering was performed based on differentially expressed mRNAs and IncRNAs using Cluster_Treeview software from Stanford University.

Transfection and gene silencing. For small interfering RNA (siRNA) transfection, the following siRNA duplexes were synthesized (Genepharma, Shanghai, China): si-Linc00963-1 (5'-GGCAAGUGCUUUCAACUCUTT-3'), and si-Linc00963-2 (5'-GCUCACUGAACUUUCUGAATT-3'), targeting the Linc00963 gene, and the negative control duplex, (5'-UUC UCCGAACGUGUCACGUTT-3'). These siRNA duplexes (100 nmol/l) were transfected into C4-2 cells using Lipofectamine 2000 (Invitrogen) according to the manufacturer's instructions. C4-2 cells were harvested $48 \mathrm{~h}$ post-transfection for gene analysis.

Real-time quantitative reverse-transcription polymerase chain reaction ( $q R T-P C R)$. Total RNA was extracted from cell lines using TRIzol reagent (Invitrogen), and subsequent synthesis of cyclic DNA (cDNA; Takara, Japan), were carried out according to the manufacturers' protocols. qRT-PCR was performed using the CFX96 ${ }^{\mathrm{TM}}$ Real-time PCR system (Bio-Rad, Hercules, CA, USA) with the SYBR Green II kit (\#DRR041A; Takara) according to the manufacturer's instructions. qRT-PCR analysis was carried out in a total volume of $20 \mu 1$ with the following amplification steps: an initial denaturation step at $95^{\circ} \mathrm{C}$ for $10 \mathrm{~min}$; followed by 40 cycles of denaturation at $95^{\circ} \mathrm{C}$ for $30 \mathrm{sec}$; and then elongation at $55^{\circ} \mathrm{C}$ for $30 \mathrm{sec}$. The expressions were normalized to the human $\beta$-actin gene. The following primer sequences were used: 5'-GGTAAATCGAGGCCCAGAGAT-3' (sense) and 5'-ACGTGGATGACAGCGTGTGA-3' (antisense) for Linc00963; 5'-CGTCTTCCCCTCCATCGT-3' (sense) and 5'-GAAGGTGTGGTGCCAGATTT-3' (antisense) for $\beta$-actin.

3-(4,5-Dimethylthiazol-2-yl)-2,5-diphenyltetrazolium bromide (MTT) assay. Cell proliferation in vitro was analyzed with MTT proliferation assay. The yellow dye MTT is reduced to a blue formazan product by respiratory enzymes that are active only in viable cells, making the amount of color change indicative of cell proliferation. C4-2 cells were transfected with no siRNA (parental), specific siRNA (si-Linc00963-1, or si-Linc00963-2) and control siRNA (si-scramble) for $24 \mathrm{~h}$ and suspended in RPMI-1640 with 10\% FBS. Briefly, 2000-3000 cells of each clone (parental, si-scramble, si-Linc00963-1 or si-Linc00963-2) were plated per well in five 96-well plates in $200 \mu \mathrm{l}$ of medium. For analysis, $20 \mu \mathrm{l}$ of MTT substrate of a 
A

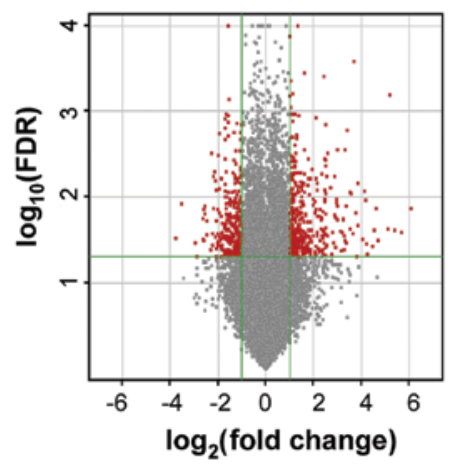

B
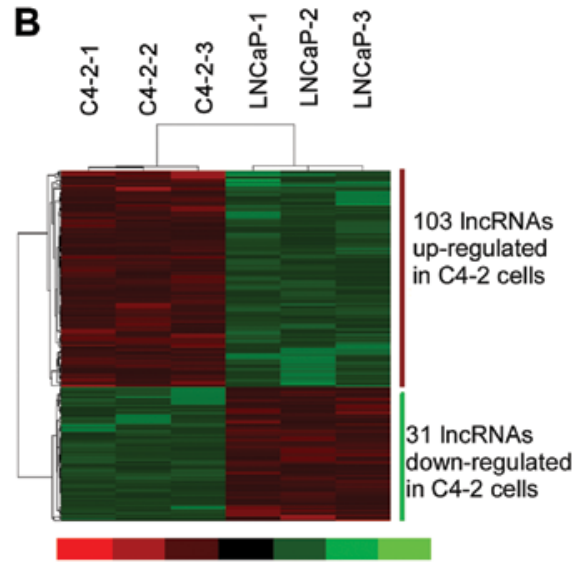

$-3.00-2.00-1.00 \quad 0 \quad 1.002 .003 .00$

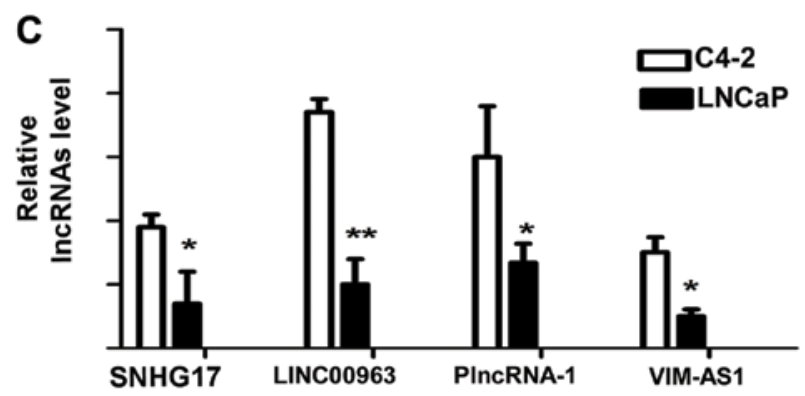

Figure 1. LncRNA is differentially expressed in the LNCaP and C4-2 cell lines. (A) Alterations in lncRNA expression profiles between C4-2 and LNCaP cell lines. The red plots represent lncRNAs which were differentially expressed (FDR $<0.05$ and fold change $\geq 2$ ) three times between LNCap and C4-2 from 46506 lincRNAs. (B) The result from Hierarchical Clustering shows distinguishable lncRNA expression profiling among samples. 'Red' indicates high relative expression; and 'Green' indicates low relative expression. (C) Linc00963 was found expressed differentially more significantly than the others in LNCaP and C4-2 cell lines.

$2.5 \mathrm{mg} / \mathrm{ml}$ stock solution in phosphate-buffered saline (PBS) was added to each well, and the cells were incubated for a further $4 \mathrm{~h}$ at $37^{\circ} \mathrm{C}$. The medium was removed, the cells were solubilized in $150 \mu \mathrm{l}$ of dimethylsulfoxide, and colorimetric analysis was performed (wavelength, $490 \mathrm{~nm}$ ). One plate was analyzed immediately after the cells adhered $(\sim 4 \mathrm{~h}$ after plating), and the remaining plates were assayed every day for the next 4 consecutive days.

Flow cytometric analysis of apoptotic cells. C4-2 cells were transfected for $48 \mathrm{~h}$ with no siRNA (parental), specific siRNA (si-Linc00963-1 or si-Linc00963-2) and control siRNA (si-scramble), then cells were suspended in incubation buffer at a density of $1 \times 10^{6}$ cells $/ \mathrm{ml}$. Apoptotic cells were analyzed by flow cytometry using a CYTOMICS FC 500 flow cytometer (Beckman Coulter), after incubating the cells with a reagent containing Annexin V-FITC and Propidium Iodide (BD Bioscience, CA, USA) for $15 \mathrm{~min}$ in darkness at room temperature.

Analysis of invasiveness and mobility (migration and invasion assays). Cell invasion and migration potentials were measured by Transwell assays (Millipore, Billerica, MA, USA). C4-2 cells were transfected for $24 \mathrm{~h}$ with no siRNA (parental), specific siRNA (si-Linc00963-1 or si-Linc00963-2) and control siRNA (si-scramble); the cells were suspended in RPMI-1640 with $8 \mathrm{~g} / \mathrm{l} \mathrm{BSA}, 10 \mathrm{mM}$ HEPES, $1.0 \mathrm{mM}$ sodium bicarbonate at a density of 50 cells $/ \mu \mathrm{l} ; 200 \mu \mathrm{l}$ cell suspensions were seeded into the upper chambers of the Transwells whose porous membrane was coated with (for Transwell invasion assay) or without (for migration assay) Matrigel (BD Bioscience). RPMI-1640 $(500 \mu \mathrm{l})$ with $8 \%$ FBS, $10 \mathrm{mM}$ HEPES, $1.0 \mathrm{mM}$ sodium bicarbonate was added to the bottom chamber as a chemoattractant. After migration for $24 \mathrm{~h}$, or invasion for $48 \mathrm{~h}$, the cells that had penetrated the filters were fixed in methanol, and stained in $4 \mathrm{~g} / \mathrm{l}$ crystal violet. The numbers of migrated and invasive cells were determined from five random fields under an Olympus microscope (Olympus) at x10 magnification.

Western blot analysis. C4-2 cells which transfected for $48 \mathrm{~h}$ with no siRNA (parental), specific siRNA (si-Linc00963-1, or si-Linc00963-2) and control siRNA (si-scramble) were harvested in radioimmunoprecipitation (RIPA) buffer. Protein concentration was determined using the BCA protein assay. Proteins were resolved using 10\% SDS-PAGE gradient gels, and $30 \mathrm{mg} /$ well was loaded. Proteins were transferred electrophoretically to PVDF membrane (Bio-Rad) at $25 \mathrm{~V}$ for $2 \mathrm{~h}$. The membrane was blocked $2 \mathrm{~h}$ at room temperature in PBS containing 5\% nonfat dry milk. Antibodies AKT and p-AKT (Bioworld Technology) were diluted in PBS-T (0.1\% Tween-20, Fisher) at 1:1000 working concentration, incubated overnight at $4^{\circ} \mathrm{C}$. The second antibody was HRP-conjugated anti-rabbit, at 1:5000 in PBS-T, and incubated for $2 \mathrm{~h}$ at room temperature. Following this, and all other incubations, membranes were washed in PBS-T 3x5 min. HRP activity was detected using ECL western blotting detection reagents. The bands were visualized by chemiluminescence (New England Nuclear, Boston, MA, USA). 
A

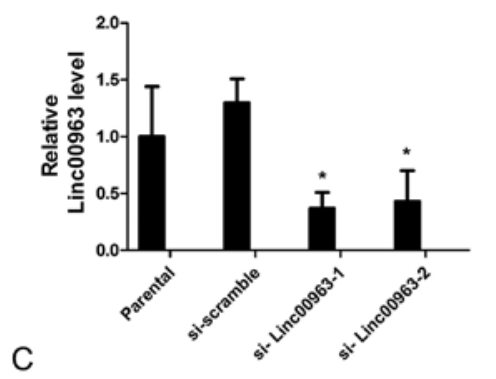

B

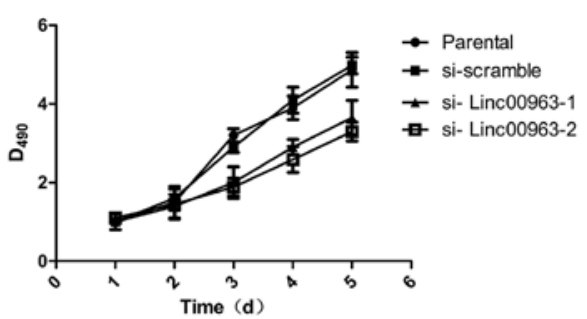

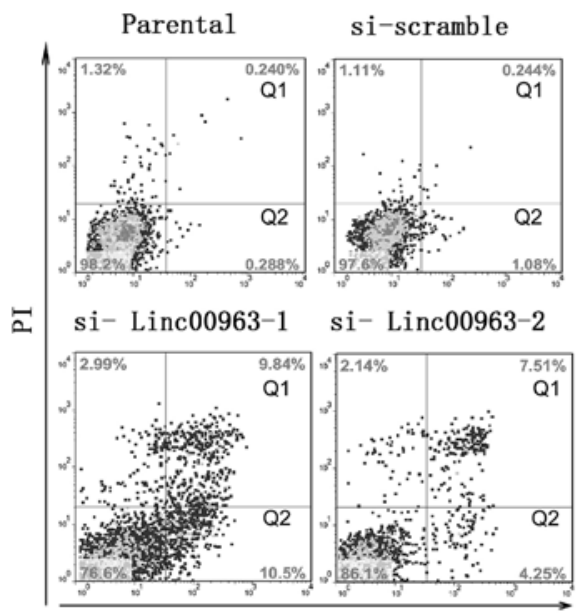

Annexin V-FITC

Figure 2. The Linc00963 knockdown affects cell viability. (A) QRT-PCR analysis of cell lines with reduced Linc00963 level after transfection with siRNAs: parental C4-2 cells carrying no siRNA (parental); scramble siRNA (si-scramble); siRNA to Linc00963 (si-Linc00963-1, si-Linc00963-2). Compared to cells transfected with scrambled siRNA, cells transfected with siRNAs targeting Linc00963 showed significantly reduced Linc00963 expression. (B) MTT cell growth assays of control cells (parental, si-scramble); cells with reduced Linc00963 expression (si-Linc00963-1, si-Linc00963-2). The results show that cells transfected with siRNAs targeting Linc00963 showed significant decrease in cell viability. Data are presented as mean $\pm \mathrm{SD}, \mathrm{N}=3$, $\mathrm{P}<0.05$. (C and D) Flow cytometric analyses of cells stained with Annexin V-FITC and PI: cells carrying no siRNA (parental), scramble siRNA (si-scramble), siRNA targeting Linc00963 (si-Linc00963-1, si-Linc00963-2). Data are presented as mean \pm SD, $N=3,{ }^{*} \mathrm{P}<0.05$. The results indicate that silencing Linc00963 expression increased cell apoptosis.

Statistical analysis. All statistical data were analyzed by Statistical Program for Social Sciences (SPSS) 20.0 software (SPSS 20.0 software, IBM, USA) and GraphPad Prism 5.0 (GraphPad Software, La Jolla, CA, USA). One-way analysis of variance test, two-tailed Student's t-test and rank-sum test were used as appropriate. $\mathrm{P}<0.05$ was considered statistically significant.

\section{Results}

Identification of the LncRNAs differentially expressed in the LNCAP and C4-2 cell lines. In order to gain better understanding of the androgen-independent and metastasis progression of prostate cancer at the lncRNA level, we generated a comprehensive IncRNA expression profile for $\mathrm{LNCaP}$ and C4-2. Total RNA was extracted from the cell lines and analyzed using microarray from Aglient to characterize the expression patterns of lncRNAs. In addition, the microarray analyses were conducted three times. The relative expression of 1ncRNAs expressed by LNCaP and C4-2 is shown in Fig. 1A, representatively. We picked out 134 lncRNAs which were differentially expressed (FDR $<0.001$ and fold change $\geq 2$ ) at three times between LNCap and C4-2 from 46506 lincRNAs represented on the chips. From the 134 lncRNAs, we identified 4 lncRNAs which were differentially expressed in LNCaP and C4-2 to the most significant extent (Fig. 1B), including the well-known lncRNA in prostate cancer, PlncRNA-1. Using RNA isolated from LNCaP and C4-2, we performed qRT-PCR to validate the expression level of the four identified lncRNAs. We found that lncRNA Linc00963 was expressed differentially most significantly compared to the others in LNCaP and C4-2 cell lines (Fig. 1C). As LNCaP is a hormone-sensitive but $\mathrm{C} 4-2$ was hormone-insensitive prostate cell line derived from LNCaP, the lncRNAs differentially expressed in $\mathrm{LNCaP}$ and $\mathrm{C} 4-2$ may be involved in the transition from AD to AI.

Effect of Linc00963 knockdown on cell viability. C4-2 is a hormone-insensitive prostate cell line and possesses the capacity of metastasizing to lymph node and bone, and our preliminary results indicated that lncRNA Linc00963 was differentially expressed in C4-2 and LNCaP significantly. Therefore, in order to investigate biological function of Linc00963 in PCa cell line C4-2, Linc00963 was suppressed by siRNAs in C4-2. The effective knockdown of Linc00963 was confirmed by qRT-PCR. Compared to cells transfected with scrambled siRNA, cells transfected with siRNAs to Linc00963 showed significantly reduced Linc00963 expres- 


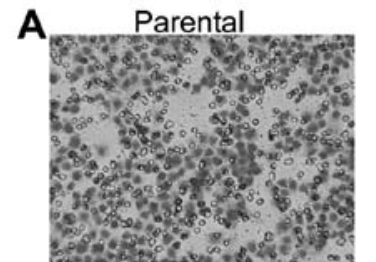

si-Linc00963-1

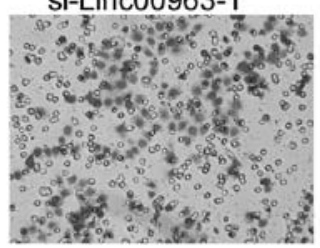

C.

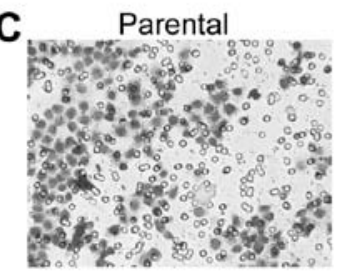

si-Linc00963-1

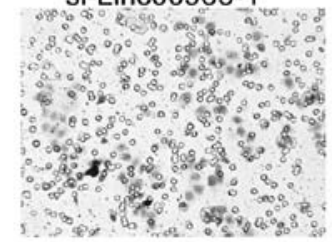

si-scramble

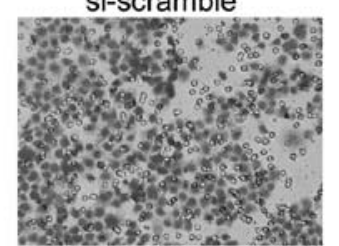

si-Linc00963-2

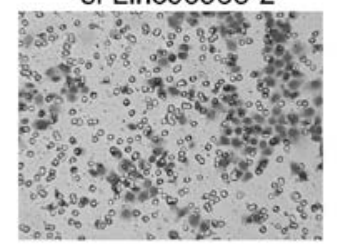

si-scramble

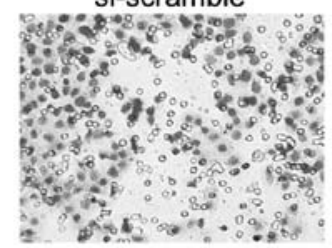

si-Linc00963-2

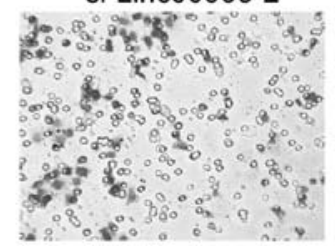

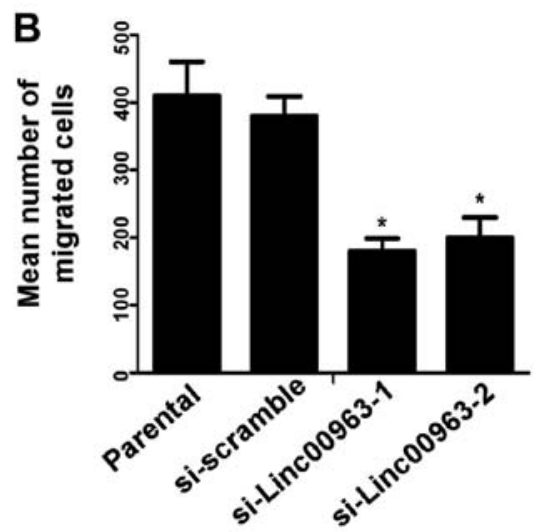

D

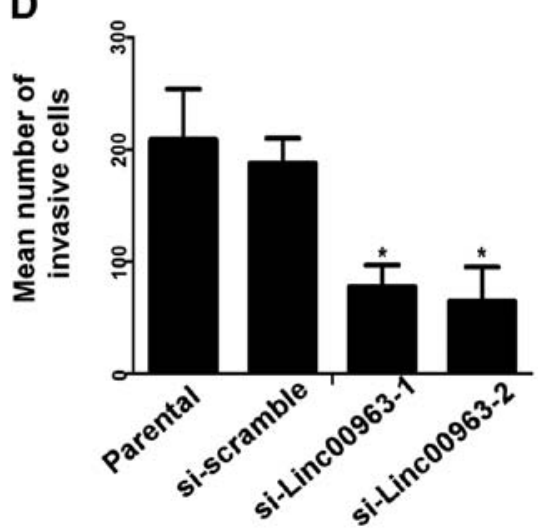

Figure 3. The Linc00963 knockdown inhibits cells migration and invasion. (A) Representative images (x10) of migration assays of C4-2 cells carrying no siRNA (parental), scramble siRNA (si-scramble), siRNA targeting Linc00963 (si-Linc00963-1, si-Linc00963-2). (B) Mean number of cells from the five independent migration assays. (C) Parental C4-2 cells, cells transfected with scramble siRNA (si-scramble), siRNA targeting Linc00963 (si-Linc00963-1, siLinc00963-2) were performed in modified Boyden chambers with Matrigel-coated membranes. After 24 h, invasive cells that had moved through the Matrigel membrane were stained and counted under a microscope at x10 magnification. (D) Graphical representation of invasive cells calculated as mean \pm SD from five fields showing statistically significantly reduced migration in siRNA transfected cells compared to control cells.

sion (each experiment was performed three times, and a typical result is presented in Fig. 2A. After confirming the knockdown efficiency of the siRNAs targeting Linc00963, we determined the effect of a reduced Linc00963 level on cell viability using an MTT assay. C4-2 cells that were transfected with siRNAs targeting Linc00963 showed significant decrease in cell viability compared to the parental or scrambled siRNAtransfected cells (Fig. 2B). This result demonstrated that the Linc00963 had a direct effect on cell viability in C4-2 cells.

Effect of the Linc00963 knockdown on cell apoptosis. To explore the mechanism by which Linc00963 affected cell vitality of C4-2, we tested whether the inhibited cell viability may be caused by increased cell apoptosis in Linc00963 knockdown cells. C4-2 cells transfected with scramble siRNA or siRNAs targeting Linc00963 for $48 \mathrm{~h}$ were analyzed for apoptosis. The results indicated that compared with the parental or scrambled siRNA-transfected cells, cells transfected with siRNAs targeting Linc00963 had increased apoptosis index which was calculated by adding the cells in the Q1 and the cells in the Q2 $(15.1 \pm 4.8 \%$ and $13.2 \pm 5.2 \%$ vs. $1.07 \pm 0.8 \%, \mathrm{P}<0.05 ; 15.1 \pm 4.8 \%$ and $13.2 \pm 5.2 \%$ vs. $0.89 \pm 0.75 \%, \mathrm{P}<0.05$; each experiment was performed three times, and a typical result is presented as Fig. 2C and D. Collectively, these results suggested that the expression of Linc00963 in C4-2 cells was important for both cell viability and apoptosis.
Effect of the Linc00963 knockdown on cell migration and invasion. To test the effects of linc00963 on migration and invasion of C4-2, we used standard Matrigel-coated or uncoated transwell chamber assays. We found that compared with the scrambled siRNA-transfected cells, C4-2 cells transfected with siRNAs targeting Linc00963 had reduced migration and invasion ability (Fig. 3A and C), and a reduced invasive index (invasion cell number/migration cell number, Fig. 3B and D). Thus, our results indicated that Linc00963 was correlated with cell migration and invasion in prostate cancer cell line C4-2.

Identification of the target molecule for Linc00963 in prostate cancer cell lines. To understand the biological function of Linc00963, the putative target sites were identified by 3 steps. Step 1: we sought to determine whether Linc00963 act in cis or in trans to regulate target gene expression. We use genome annotation, genome browser and RNAplex to find these putative targets (Fig. 4A). Step 2: unsupervised hierarchical clustering was used to analyze the differential mRNA expression profiles. Among these candidate targets, we identified 5 genes, ARHGEF26, EGFR, HYAL1, ICAM1 and PPFIA2 as the putative targets, which were differentially expressed in C4-2 and LNCaP cells to the most significant extent and may be regulated by Linc00963 through transcriptional interference (Table I). Step 3: after identification of these targets, we further validated our results in the prostate cell lines 

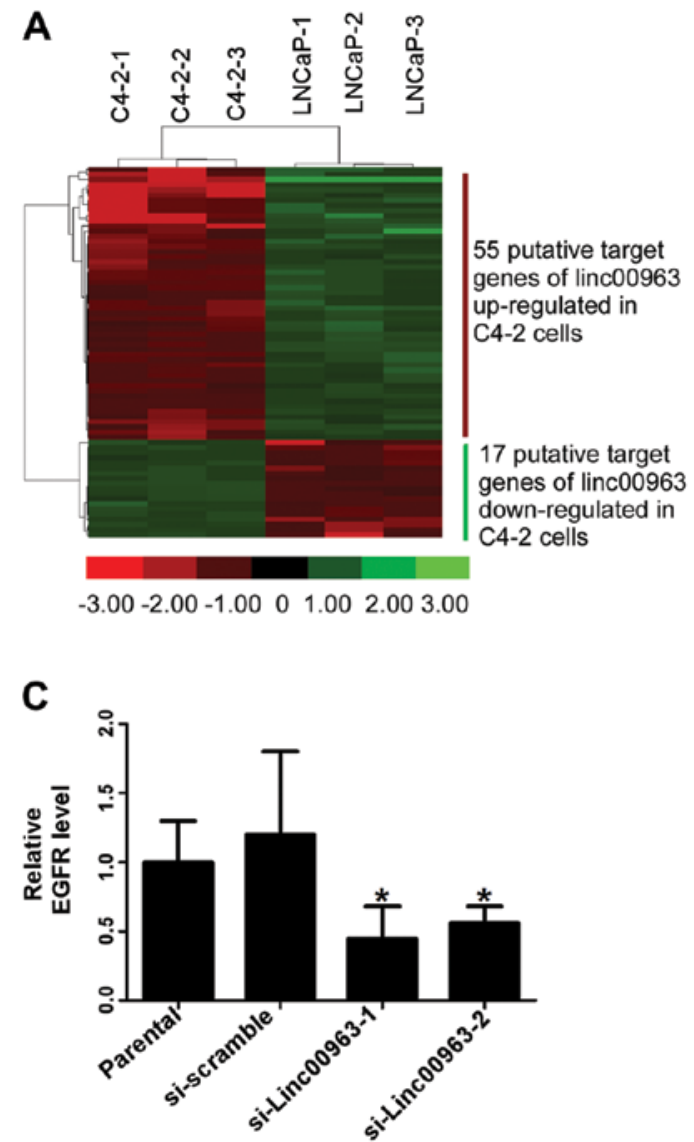

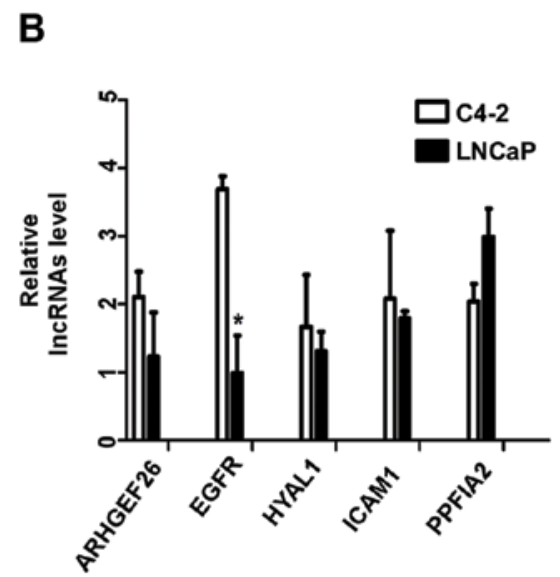

D

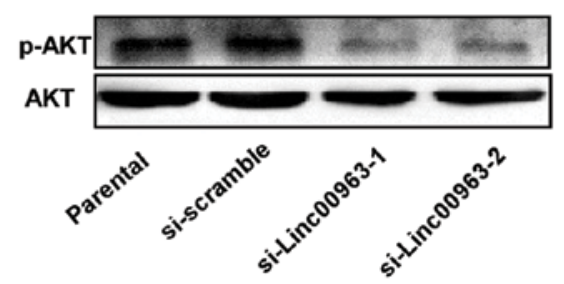

Figure 4. EGFR was the putative target molecule of Linc00963 in prostate cancer cells. (A) Heat map of the Linc00963 putative targets. (B) EGFR is the most likely putative target of Linc00963. (C) Knockdown of Linc00963 significantly decreases EGFR level. (D) The phosphorylation level of AKT, the downstream gene of EGFR, decreased after silencing of Linc00963.

LNCaP and C4-2. From the results of qRT-PCR, we found EGFR was expressed most differentially in LNCaP and C4-2 (Fig. 4B). To investigate whether EGFR was the putative target for Linc00963, we performed qRT-PCR to examine the expression level of EGFR in C4-2 cells transfected with Linc00963 siRNAs. In addition, we tested the phosphorylation level of its downstream molecule AKT which has been proven to correlate with castration resistant cell growth and androgen receptor level in CRPC (25). Our results showed knockdown of Linc00963 significantly decreases EGFR, and the phosphorylation level of AKT (Fig. 4C and D), indicating Linc00963 involved in the transactivation of EGFR in hormone-insensitive prostate cancer cells. In conclusion, our result indicated that EGFR was the target molecule of Linc00963 in prostate cancer cells.

\section{Discussion}

Progression to androgen resistance and metastasis of $\mathrm{PCa}$, involve alterations in gene expression and dysregulation of signaling pathways, and remains both an intensive and elusive area of investigation. In addition to protein coding genes and miRNAs, dysregulatory of lncRNAs is emerging as a ubiquitous component in the gene regulatory networks of cancer progression $(11,26)$. In the current study, for the first time, lncRNA Linc00963 (GeneBank accession ID: 100506190) is found to be involved in the progression from $\mathrm{AD}$ to $\mathrm{AI}$ of prostate cancer. We characterized Linc00963, which is more frequently overexpressed in hormone-insensitive prostate cell line $\mathrm{C} 4-2$ than hormone-sensitive prostate cell line LNCaP, and its overexpression correlated with cell viability, cell apoptosis, cell migration and cell invasion of C4-2, suggesting an important role of Linc00963 in the transition from AD to AI.

Previous studies showed that lincRNAs could act in cis (on neighboring genes) by transcriptional interference and/or function in trans (on distant located genes) though targeting epigenetic modifiers $(27,28)$. We identified that Linc00963 and EGFR were located in different chromosomes and Linc00963 had a functional relationship with EGFR, so we supposed Lin00963 might act in trans with EGFR. We further confirmed that EGFR and the phosphorylation level of its downstream gene AKT decreased following knockdown of Linc00963. These results demonstrated that EGFR was the putative target molecule of Linc00963 and Linc00963 involved in the transactivation activity of EGFR in prostate cancer cells. However, further studies are required to clarify the molecular mechanism underlying the regulation of Linc00963 and EGFR.

LncRNAs are being recognized at every level of gene expression in various physiological processes, and alteration of the expression of IncRNAs in cancer is considered as one of the main driving forces during tumorigenesis $(26,29,30)$. In prostate cancer, the function of lncRNAs is more complex 
Table I. The potential target molecular for Linc00963 in prostate cancer cell lines.

Gene name

\begin{tabular}{llr}
\hline ATG9B & & 3.123 \\
ADAMTS1 & Autophagy related 9B & 3.735 \\
ANKRD18A & ADAM metallopeptidase with thrombospondin type 1 motif, 1 & 3.178 \\
ARHGEF26 & Ankyrin repeat domain 18A & 4.416 \\
AZGP1 & Rho guanine nucleotide exchange factor (GEF) 26 & $1 / 3.576$ \\
GPR158 & $\alpha$-2-glycoprotein 1, zinc-binding & 3.186 \\
HES1 & G protein-coupled receptor 158 & $1 / 3.314$ \\
HYAL1 & Hes family bHLH transcription factor 1 & 5.643 \\
ICAM1 & Hyaluronoglucosaminidase 1 & 4.176 \\
KLK2 & Intercellular adhesion molecule 1 & $1 / 3.591$ \\
KLK3 & Kallikrein-related peptidase 2 & $1 / 3.332$ \\
PIK3R1 & Kallikrein-related peptidase 3, prostate specific antigen & 3.611 \\
PPFIA2 & Phosphoinositide-3-kinase, regulatory subunit 1 $(\alpha)$ & $1 / 4.872$ \\
& Protein tyrosine phosphatase, receptor type, f polypeptide (PTPRF), \\
PRSS2 & interacting protein (liprin), $\alpha 2$ & 3.331 \\
S100A10 & Protease, serine, 2 (trypsin 2) & $1 / 3.012$ \\
Nrp1 & S100 calcium binding protein A10 & 3.277 \\
NUCB2 & Neuropilin 1 & 3.638 \\
MYLK & Nucleobindin 2 & $1 / 3.547$ \\
EGFR & Myosin light chain kinase & 5.347 \\
ELF3 & Epidermal growth factor receptor & $1 / 3.326$ \\
ELOVL5 & E74-like factor 3 (ets domain transcription factor, epithelial-specific) & 3.936 \\
GDF11 & ELOVL fatty acid elongase 5 & $1 / 3.176$ \\
GNAI1 & Growth differentiation factor 11 & $1 / 3.195$ \\
GAS6 & Guanine nucleotide binding protein (G protein), $\alpha$ inhibiting & 3.079 \\
\hline
\end{tabular}

Fold change expression C4-2/LNCaP than previously believed. More and more lncRNAs were found dysregulated in prostate cancer, and most of them exhibit oncogenic function, including prostate cancer antigen 3 (PCA3) (31), PCGEM1, PRNCR1, MALAT-1, PlncRNA-1 (32), and CTBP1-AS (33). Most of these identified lncRNAs was proven to be associated with androgen receptor (AR), but no previous studies concerning lncRNAs paid attention to the mechanism that would account for the transition from AD to AI. Thus, we used microarray technologies to delineate the differential expression profiles of cancer-related lncRNAs in hormone-sensitive and hormone-insensitive prostate cancer cell lines. RNA-seq revealed that 134 lncRNAs were expressed differentially in LNCaP and C4-2, and Linc00963 was upregulated to the most significant extent in C4-2 among these dysregulated lncRNAs. These results led us to believe that Linc00963 is potentially involved in the progeression of AD PCa to the lethal AI phenotype. To our knowledge, this is the first study using microarray technologies to delineate the IncRNA profiles between LNCaP and C4-2 cells.

Castration-resistant prostate cancer (CRPC) tends to progress and metastasize, and shows short survival. The prostate cancer cell line C4-2 acquires the phenotypes of androgenindependence and osseous metastases, and it is commonly used in models of castration-resistant and aggressive prostate cancer (22). The second part of our study identified the role of Linc00963 in AI prostate cancer cells. Our results indicated that Linc00963 is significantly associated with the cell viability, cell motility and cell invasiveness. Although a more detailed mechanism must be discovered to explain our results, these results provide evidence that Linc00963 may function as an oncogenic molecule and linc0096 is a ubiquitous component in the gene regulatory networks of prostate cancer development and progression. Though previous studies had identified some lncRNAs aberrantly expressed exhibiting oncogenic function in $\mathrm{PCa}$, such as inhibiting apoptosis or promoting cell proliferation $(10,16,17,21)$, no direct evidence was provided indicating the role of lncRNAs in AI prostate cancer development. Our lncRNA expression profiling results and the effect of Linc00963 on tumor metastasis in LNCaP and C4-2 partially illustrate the role InRNAs played in the transition from $\mathrm{AD}$ to $\mathrm{AI}$ and the progress of AI prostate cancer metastasis. 
The third part of our study identified EGFR as a putatively functional target of Linc00963 in the hormone-insensitive prostate cancer cell line C4-2. Enhanced expression of EGFR/ErbB1 had been proven to correlate with high grades of prostate cancer malignancies and contribute to the progression from localized and AD prostate cancer to more metastatic and AI state $(34,35)$, and the phosphorylation level of AKT which was the downstream gene of EGFR had been proven to correlate with androgen receptor level and promote castration- resistant cell growth (25). Importantly, AR was observed to be slightly downregulated in C4-2 (36). Taken together, all these studies indicated EGFR might play a more important role in the process from AD to AI than AR. However, most of the well characterized prostate cancer-related lncRNAs were proved to be closely associated with AR, which indicated these lncRNAs played secondary role in the tansition from AD to AI. Whereas, we infer that IncRNAs, which were correlated with EGFR, might play a pivotal role in the progression of $\mathrm{PCa}$.

Although we identified lncRNAs as a ubiquitous component in the gene regulatory networks of prostate cancer progression, more work need to be done to eventually clarify the underlying mechanism mediating the transition from AD to AI. The most important aspect is to make clear the interaction between IncRNAs and EGFR. Our results indicated that the lncRNA Linc00963 could affect the expression level of EGFR at transcriptional level, but we did not obtain any evidence to support their direct interaction between EGFR protein complex and Linc00963. The second limitation of our study is we confirmed the role of lincRNAs in hormone-sensitive and hormone-insensitive prostate cancer cell line LNCaP and C4-2, but we did not further validate our result in the tissues obtained from $\mathrm{AD}$ and AI prostate cancer patients. Although use of LNCaP and C4-2 cell line is an accepted model for studying the mechanism underlying the progression of PCa from $\mathrm{AD}$ to $\mathrm{AI}$, the confirmation from experiments in clinical specimens will make our results more conclusive and convincing. These limitations indicate that more detailed work is required to clarify the relationship between lincRNAs and EGFR, to further improve our knowledge concerning the transition from $\mathrm{AD}$ to $\mathrm{AI}$ in prostate cancer.

In summary, we have identified that the long intergenic non-protein coding RNA 00963 is upregulated in hormoneinsensitive prostate cancer cell line C4-2 but downregulated in hormone-sensitive prostate cancer cell line LNCaP. The knockdown of Linc00963 in hormone-insensitive prostate cancer cells inhibits cell viability, motility and invasiveness. Linc00963 physically associates with EGFR and could be an important regulator for the transition in prostate cancer from AD to AI. Collectively, our data provide insight into molecular characteristics of AI and metastatic prostate cancer and provide clues for finding new strategies to prevent PCa metastasis.

\section{Acknowledgements}

This study was supported by the National Natural Science Foundation of China (no. 81272200, 81072117).

\section{References}

1. Siegel R, Naishadham D and Jemal A: Cancer statistics, 2013. CA Cancer J Clin 63: 11-30, 2013.
2. Lee AK: Radiation therapy combined with hormone therapy for prostate cancer. Semin Radiat Oncol 16: 20-28, 2006.

3. Molina A and Belldegrun A: Novel therapeutic strategies for castration resistant prostate cancer: inhibition of persistent androgen production and androgen receptor mediated signaling. J Urol 185: 787-794, 2011.

4. Mlcochova J, Faltejskova P, Nemecek R, Svoboda M and Slaby O: MicroRNAs targeting EGFR signalling pathway in colorectal cancer. J Cancer Res Clin Oncol 139: 1615-1624, 2013.

5. Shuch B, Mikhail M, Satagopan J, et al: Racial disparity of epidermal growth factor receptor expression in prostate cancer. J Clin Oncol 22: 4725-4729, 2004.

6. Syed S: Combination chemotherapy for hormone-refractory prostate carcinoma: progress and pitfalls. Cancer 98: 2088-2090, 2003.

7. Traish AM and Morgentaler A: Epidermal growth factor receptor expression escapes androgen regulation in prostate cancer: a potential molecular switch for tumour growth. Br J Cancer 101: 1949-1956, 2009

8. Antonarakis ES, Carducci MA and Eisenberger MA: Novel targeted therapeutics for metastatic castration-resistant prostate cancer. Cancer Lett 291: 1-13, 2010.

9. Guttman M, Amit I, Garber M, et al: Chromatin signature reveals over a thousand highly conserved large non-coding RNAs in mammals. Nature 458: 223-227, 2009.

10. Prensner JR and Chinnaiyan AM: The emergence of lncRNAs in cancer biology. Cancer Discov 1: 391-407, 2011.

11. Gupta RA, Shah N, Wang KC, et al: Long non-coding RNA HOTAIR reprograms chromatin state to promote cancer metastasis. Nature 464: 1071-1076, 2010.

12. Reis EM, Nakaya HI, Louro R, et al: Antisense intronic noncoding RNA levels correlate to the degree of tumor differentiation in prostate cancer. Oncogene 23: 6684-6692, 2004.

13. Prensner JR, Iyer MK, Balbin OA, et al: Transcriptome sequencing across a prostate cancer cohort identifies PCAT-1, an unannotated lincRNA implicated in disease progression. Nat Biotechnol 29: 742-749, 2011.

14. Ji P, Diederichs S, Wang W, et al: MALAT-1, a novel noncoding RNA, and thymosin beta4 predict metastasis and survival in early-stage non-small cell lung cancer. Oncogene 22: 8031-8041, 2003.

15. Petrovics G, Zhang W, Makarem M, et al: Elevated expression of PCGEM1, a prostate-specific gene with cell growth-promoting function, is associated with high-risk prostate cancer patients. Oncogene 23: 605-611, 2004.

16. Srikantan V, Zou Z, Petrovics G, et al: PCGEM1, a prostatespecific gene, is overexpressed in prostate cancer. Proc Natl Acad Sci USA 97: 12216-12221, 2000.

17. Chung S, Nakagawa H, Uemura M, et al: Association of a novel long non-coding RNA in 8q24 with prostate cancer susceptibility. Cancer Sci 102: 245-252, 2011.

18. Cui Z, Ren S, Lu J, et al: The prostate cancer-up-regulated long noncoding RNA PlncRNA-1 modulates apoptosis and proliferation through reciprocal regulation of androgen receptor. Urol Oncol 31: 1117-1123, 2013.

19. Lin R, Maeda S, Liu C, Karin M and Edgington TS: A large noncoding RNA is a marker for murine hepatocellular carcinomas and a spectrum of human carcinomas. Oncogene 26: 851-858, 2007.

20. de Kok JB, Verhaegh GW, Roelofs RW, et al: DD3(PCA3), a very sensitive and specific marker to detect prostate tumors. Cancer Res 62: 2695-2698, 2002.

21. Hessels D, Klein Gunnewiek JM, van Oort I, et al: DD3(PCA3)-based molecular urine analysis for the diagnosis of prostate cancer. Eur Urol 44: 8-16, 2003.

22. Xie BX, Zhang $\mathrm{H}, \mathrm{Yu} \mathrm{L}$, et al: The radiation response of androgen-refractory prostate cancer cell line C4-2 derived from androgen-sensitive cell line LNCaP. Asian J Androl 12: 405-414, 2010.

23. Horoszewicz JS, Leong SS, Kawinski E, et al: LNCaP model of human prostatic carcinoma. Cancer Res 43: 1809-1818, 1983.

24. Thalmann GN, Anezinis PE, Chang SM, et al: Androgenindependent cancer progression and bone metastasis in the LNCaP model of human prostate cancer. Cancer Res 54: 2577-2581, 1994

25. Chen L, Mooso BA, Jathal MK, et al: Dual EGFR/HER2 inhibition sensitizes prostate cancer cells to androgen withdrawal by suppressing ErbB3. Clin Cancer Res 17: 6218-6228, 2011.

26. Gutschner T and Diederichs S: The hallmarks of cancer: a long non-coding RNA point of view. RNA Biol 9: 703-719, 2012. 
27. Wang $\mathrm{KC}$ and Chang HY: Molecular mechanisms of long noncoding RNAs. Mol Cell 43: 904-914, 2011.

28. Ponting CP, Oliver PL and Reik W: Evolution and functions of long noncoding RNAs. Cell 136: 629-641, 2009.

29. Roberts LD, Murray AJ, Menassa D, Ashmore T, Nicholls AW and Griffin JL: The contrasting roles of PPARdelta and PPARgamma in regulating the metabolic switch between oxidation and storage of fats in white adipose tissue. Genome Biol 12: R75, 2011.

30. Cheng W, Zhang Z and Wang J: Long noncoding RNAs: New players in prostate cancer. Cancer Lett 339: 8-14, 2013.

31. Bussemakers MJ, van Bokhoven A, Verhaegh GW, et al: DD3: a new prostate-specific gene, highly overexpressed in prostate cancer. Cancer Res 59: 5975-5979, 1999.

32. Bu D, Yu K, Sun S, et al: NONCODE v3.0: integrative annotation of long noncoding RNAs. Nucleic Acids Res 40: D210-D215, 2012.
33. Takayama K, Horie-Inoue K, Katayama S, et al: Androgenresponsive long noncoding RNA CTBP1-AS promotes prostate cancer. EMBO J 32: 1665-1680, 2013.

34. Mimeault $M$ and Batra SK: Recent advances on multiple tumorigenic cascades involved in prostatic cancer progression and targeting therapies. Carcinogenesis 27: 1-22, 2006.

35. Nickerson T, Chang F, Lorimer D, Smeekens SP, Sawyers CL and Pollak M: In vivo progression of LAPC-9 and LNCaP prostate cancer models to androgen independence is associated with increased expression of insulin-like growth factor I (IGF-I) and IGF-I receptor (IGF-IR). Cancer Res 61: 6276-6280, 2001.

36. Xie BX, Zhang H, Wang J, et al: Analysis of differentially expressed genes in $\mathrm{LNCaP}$ prostate cancer progression model. $\mathrm{J}$ Androl 32: 170-182, 2011. 